\title{
Relationship between Obesity and UTI in Children Under 2 Years of Age Admitted with Fever
}

\author{
SuYeong Ko, M.D. \\ Jae Hee Lee, M.D. \\ Young II Rho, M.D. \\ Department of Pediatrics, Chosun \\ University School of Medicine, \\ Gwangju, Korea \\ *This study was supported by research \\ fund from Chosun University 2017. \\ Corresponding author: \\ Young II Rho, M.D. \\ Department of Pediatrics, Chosun \\ University School of Medicine, 365 \\ Pilmon-daero, Dong-gu, Gwangju 61453, \\ Korea \\ Tel: $+82-62-220-3036$ \\ Fax: +82-62-227-2904 \\ E-mail: ryoung@chosun.ac.kr
}

Received: 14 September 2018

Revised: 4 October 2018

Accepted: 12 October 2018

\begin{abstract}
This is an open-access article distributed under the terms of the Creative Commons Attribution Non-Commercial License (http:// creativecommons.org/licenses/by-nc/4.0/) which permits unrestricted non-commercial use, distribution, and reproduction in any medium, provided the original work is properly cited.
\end{abstract}

Purpose: We observed an association between obesity and UTI in infants and pediatric patients, which we aimed to validate in this study.

Method: The medical records of 740 patients ( $\leq 24$ months old) hospitalized with fever were retrospectively analyzed. The patients were subdivided into 2 groups, namely, the UTI and control (non UTI) groups. We analyzed the patient's height, weight. Obesity was defined as weight-for-length $\geq 95^{\text {th }}$ percentile, and the association between obesity and UTI was evaluated.

Results: Out of 740 patients, 253 and 487 patients were in the UTI group and the control group, respectively. A comparative analysis, based on 3 age groups $(0-5$ months, 6-11 months, and 12-24 months) showed higher proportion of obesity in the UTI group (26.4\%) than in the control group (13.0\%) in the 0-5months group. After adjusting for age and gender in the $0-5$ month group, the obesity group was 3.76 times likely to have an UTI ( $95 \% \mathrm{Cl} 1.419-9.98)$.

Conclusion: Obesity and UTI show strong association, especially in infants aged 0-5 months. Febrile obese infant patients ( $\leq 5$ months old) visiting medical centers should be advised urine tests for potential UTI.

Key words: Urinary tract infection, Pediatrics, Obesity

\section{Introduction}

Urinary tract infection (UTI) in infants is not manifested by specific signs or symptoms, with fever often being the only $\operatorname{sign}^{1,2)}$. Therefore, UTI cannot be accurately diagnosed, based on only the symptoms or the physical examination. The gold standards for the diagnosis of UTI are urinalysis and a positive urine culture test. UTI requires early treatment as it can cause renal scarring, hypertension, and chronic renal failure if not treated appropriately ${ }^{3)}$.

The risk factors for UTI include age, circumcision status in boys, obstruction of the urinary tract, vesicoureteral reflux, and voiding dysfunction. Obesity is controversial as a risk factor ${ }^{4,5)}$.

Recent studies have reported an association between obesity and UTI in both adult and pediatric patients. Studies on adult patients reported that a higher body-mass index (BMI) was associated with an increased risk of UTI 6). A few other studies on pediatric patients were reported. Yang et al. reported that a higher proportion of overweight and obese patients was observed in pediatric patients ( $<3$ years old) diagnosed with UTI than in healthy controls 
${ }^{7)}$. Although studies have compared the percentage of obesity between healthy control groups and UTI groups, to the best of our knowledge, no study has been conducted on febrile young children without UTI. The present study assesses the hypothesis that an increased risk for UTI is observed in febrile pediatric patients ( $\leq 24$ months old) who are obese than in febrile children with normal weight-forlength. The main purpose of our study was to elucidate whether obesity is a contributing factor to the occurrence of UTIs in young children.

\section{Materials and methods}

We retrospectively reviewed the medical records of 740 young febrile children below the age of 24 months in the department of pediatrics, Chosun University Hospital, between January 2010 and December 2016. The control group consisted of febrile children without UTI. The criteria for inclusion of patients with UTIs was fever, pyuria, and a positive urine culture with pure growth of $>10^{5}$ organisms $/ \mathrm{mL}$ on a catheterized urine. Demographic factors such as gender, age, feeding pattern (only in the infants of $\leq 5$ months in age), and history of UTI were collected for individuals who met the inclusion criteria. The weight and height were determined using a digital electronic scale with the children in lightweight clothing. All patients were subcategorized into 3 groups as follows: lean (weight-for-length $<85^{\text {th }}$ percentile), overweight $\left(85^{\text {th }} \leq\right.$ weight-for-length $<95^{\text {th }}$ percentile), and obese (weight-for-length $\geq 95^{\text {th }}$ percentile), based on another report that identified obesity in young children ${ }^{7,8)}$.

Laboratory measurements in patients with UTIs included WBC counts, C-reactive protein levels, urinalysis, and urine culture. Renal sonograms and technetium-99mlabeled dimercaptosuccinicacid (DMSA) scans were performed within 5 days of the initial UTI diagnosis. If focal or diffuse diminished uptake was demonstrated on the DMSA scan, the patient was diagnosed with acute pyelonephritis (APN). Voiding cystourethrography (VCUG) was performed 3 weeks after the diagnosis of UTI.

In this study, we subdivided the patients into 3 groups based on obesity and assessed the relationship between the UTI group and control group. In addition, based on pre- vious studies assessing the association between obesity and breastfeeding method, we also aimed to assess the relationship between breastfeeding method and $\mathrm{UTI}^{9-12)}$.

All statistical analyses were performed using SPSS 24.0 (SPSS Inc, Chicago). Categorical variables were assessed using chi-square test and Fisher exact test. Continuous variables that were normally distributed were compared using the Student's t-test and that were not normally distributed were compared using the Mann-Whitney U test. Multiple logistic regression model analysis was used to assess the association between UTI and weight-for-length category after adjusting for age and gender. A $P$ value of less than 0.05 for the 2-tailed test was considered statistically significant.

The study protocol was approved by the institutional board, with permission to review the electronic medical records. (IRB no. 2018-04-014)

\section{Results}

There were 253 patients in the UTI group comprising 186 male patients (73.5\%) and 67 female patients (26.5\%). The control group was composed of 487 patients, which included 348 patients with acute pharyngitis, 51 patients with acute bronchitis, 43 patients with pneumonia, and 20 patients with fever of unknown origin. Although there were 348 patients with acute pharyngitis, the origin of fever in these patients was unclear in most cases. There were 284 male patients (58.3\%) and 203 female patients (41.7\%) in the control group. Although both groups had more males than females, the ratio was significantly higher in the UTI group $(P<0.001)$. The median age was 2.0 months in the UTI group, which was significantly lower than the median age of 13.0 months in control group $(P<0.001)$.

Because of the difference in median age, the patients were further subdivided based on their ages ( $0-5$ months, 6-11 months, and 12-24 months) for the comparison between the UTI group and the control group. In the group aged 05 months, both the UTI group and the control group had same median age (2 months). Although the UTI group had a greater number of males (154 patients, $74.0 \%$ ) than the control group (48 patients, $62.3 \%$ ), the difference was not statistically significant $(P=0.054)$. There were 55 obese pa- 
tients (26.4\%) and 19 overweight patients (9.1\%) in the UTI group, and 10 obese patients (13.0\%) and 13 overweight patients (16.9\%) in the control group. The proportion of patients with obesity was significantly higher in the UTI group than in the control group $(P=0.021)$ (Table 1). According to the multivariate analysis adjusted for age and gender, the proportion of obesity in the UTI group was higher than that in the control group. $(P=0.028)$. The obesity group was 3.76 times likely to have an UTI (95\% CI 1.419-9.98) (Table 2).

In the 6-11 months group, there were 4 patients with obesity (10.8\%) and 5 overweight patients (13.5\%) in the UTI group, whereas in the control group, 5 patients were obese

Table 1. Comparison of UTI Group and Control Group in the Age of $0-5$ Months

\begin{tabular}{lccc}
\hline & UTI $(n, \%)$ & Control $(n, \%)$ & $P$ value \\
\hline Age (months) & 2.0 & 2.0 & 0.574 \\
Sex & & & 0.054 \\
Male & $154(74.0 \%)$ & $48(62.3 \%)$ & \\
Female & $54(26.0 \%)$ & $29(37.7 \%)$ & \\
Weight-for-length & & & 0.021 \\
Obesity & $55(26.4 \%)$ & $10(13.0 \%)$ & \\
Overweight & $19(9.1 \%)$ & $13(16.9 \%)$ & \\
Lean & $134(64.5 \%)$ & $54(70.1 \%)$ & \\
Total & $208(100 \%)$ & $77(100 \%)$ & \\
\hline
\end{tabular}

Table 2. Multivariate Analysis for the Probability of UTI at the Age of $0-5$ Months

\begin{tabular}{lccc}
\hline & OR & $95 \% \mathrm{Cl}$ & $P$ value \\
\hline Age & 1.717 & $0.974-3.028$ & 0.062 \\
Weight-for-length & & & 0.028 \\
(Obesity) & 3.763 & $1.419-9.982$ & \\
\hline
\end{tabular}

Abbreviations: UTI, urinary tract infection; $\mathrm{OR}$, odd ratio; $\mathrm{Cl}$, confidence interval.
(3.5\%) and 18 patients (12.9\%) were overweight. Although the proportions of patients with obesity or overweight patient were higher in the UTI group, the difference was not significant $(P=0.99)$. No meaningful results were found in the 12-24 months group (Table 3).

After excluding 3 patients out of 285 infant patients ( $\leq 5$ months old) from both groups due to missing information regarding breastfeeding methods, the breastfeeding patterns of 282 patients were analyzed. Among the UTI group (205 infants), 46 patients (22.4\%) were exclusively breast feeding, and 159 patients (77.6\%) were formula feeding (including mixed feeding). And among the control group (77 infants), 24 patients (31.1\%) were exclusively breast feeding, and 53 patients (68.9\%) were formula feeding. Although the percentage of breast feeding in the UTI group (22.4\%) was lower than the value of the control group (31.3 $\%)$, the difference was not significant $(P=0.178)$.

We compared the renal sonogram, VCUG, DMSA scan, and laboratory measurements for the weight-for-length in the UTI group, but did not yield a meaningful result (Table $4)$.

\section{Discussion}

In our study, the rate of obesity in the aged 0-5 months of UTI patients was $26.4 \%$, higher than the control group of febrile patients without UTI (13.0\%). Previous studies that compared healthy children and pediatric UTI patients showed an association between UTI and being overweight or obese. Yang et al., in their study of pediatric patients $(<3$ years old), reported that the rate of overweight and obese

Table 3. Comparison of UTI Group and Control Group in the Age of 6-11 Months and 12-24 Months

\begin{tabular}{|c|c|c|c|c|c|c|}
\hline & \multicolumn{3}{|c|}{ 6-11 months } & \multicolumn{3}{|c|}{ 12-24 months } \\
\hline & UTI $(n, \%)$ & Control $(n, \%)$ & $P$ value & UTI $(n, \%)$ & Control $(n, \%)$ & $P$ value \\
\hline Age (months) & 7.0 & 9.0 & $<0.001$ & 15.0 & 17.0 & 0.547 \\
\hline Sex & & & 0.068 & & & 1.000 \\
\hline Male & 27 (73.0\%) & 79 (56.4\%) & & 5 & 157 & \\
\hline Female & $10(27.0 \%)$ & 61 (43.6\%) & & 3 & 113 & \\
\hline Weight-for-length & & & 0.994 & & & 0.619 \\
\hline Obesity & $4(10.8 \%)$ & 15 (10.7\%) & & 0 & $11(4.0 \%)$ & \\
\hline Overweight & $5(13.5 \%)$ & $18(12.6 \%)$ & & 0 & $18(6.7 \%)$ & \\
\hline Lean & 28 (75.7\%) & 107 (76.4\%) & & $8(100 \%)$ & 241 (89.3\%) & \\
\hline Total & 37 (100\%) & 140 (100\%) & & $8(100 \%)$ & 270 (100\%) & \\
\hline
\end{tabular}


patients was higher in febrile UTI and APN groups than in the healthy control group ${ }^{7}$. Furthermore, pediatric UTI patients with obesity exhibited a longer duration of fever and a worse diagnosis of APN. Semins et al. showed that the diagnosis of pyelonephritis was 5 times greater in obese patients than in non-obese patients, and the incidence correlates with increasing values of $\mathrm{BMI}^{13)}$. Even though our study limited the age range of the patients further ( $\leq 2$ years old) and compared UTI versus non-UTI febrile children instead of UTI versus a healthy control group, we observed an identical association between obesity and UTI.

More specifically, in our study, the association between obesity and UTI was confirmed to be a meaningful factor only inpatients aged $0-5$ months. The obese group was more likely to have an UTI by 3.76 fold. In pediatric patients of $\leq 24$ months in age, a fever $\left(>39^{\circ} \mathrm{C}\right)$ without an apparent source is the most common sign of urinary tract infection ${ }^{14)}$. Urine collection in children who are not toilettrained requires urethral catheterization or suprapubic aspirate. However, these methods are not realistic options in outpatient clinic settings and may also burden the pediatric patients, and therefore are rarely used. According to the association between obesity and UTI identified in our study, we believe that urine tests using these methods should be performed in febrile pediatric patients who are $\leq 5$ months old.
The urinary tract is closely located to the fecal flora but is sterile, and the mechanism behind this phenomenon remains unclear. The host immune response plays an important role in protecting the urinary tract against microbial insult. When a microbial insult occurs, uroepithelial toll like receptors 4 (TLR4) induces cascade of signal to activate pro-inflammatory pathway ${ }^{15}$. However, obese patients exhibit aberrant immune response and have increased dysfunction and production of adipokines in the T-cells and macrophages. This, in turn, causes inflammatory cytokine levels to be higher in general, which consequently induces a chronic inflammatory state and weakens the immune response against pathogens, exposing the host to infection ${ }^{16)}$. Moreover, in an experimental study on rats, Yim et al. stated that since obesity increased the incidence of renal inflammation and progressive kidney disorders, it elevated the risk of UTI and acute pyelonephritis ${ }^{17,18)}$. Yang et al. claimed that increased sympathetic activity associated with obesity can increase the prostate volume, affect voiding dysfunction, and consequently increase the risk of $\mathrm{UTI}^{7}$.

Unlike previous reports, in our study, the association between obesity and UTI was confirmed to be a meaningful factor only in the 0-5 months old group and not in the 624 months old group. The reason behind this observation could not be identified. In a previous Scottish birth cohort (1997-2009) study that assessed the association between

Table 4. Comparison of Lean, Overweight and Obesity in the UTI Groups

\begin{tabular}{|c|c|c|c|c|c|}
\hline & Lean & Overweight & Obesity & Total & $P$ value \\
\hline Age (months) & 2.5 & 2.5 & 2 & & 0.325 \\
\hline Sex & & & & & 0.053 \\
\hline Male & 131 & 13 & 42 & 186 & \\
\hline Female & 39 & 11 & 17 & 67 & \\
\hline Renal sonogram & & & & & 0.941 \\
\hline Hydronephrosis, n (\%) & $59(67.0 \%)$ & $9(10.2 \%)$ & $20(22.8 \%)$ & $88(100 \%)$ & \\
\hline Normal, n(\%) & $105(66.0 \%)$ & 15 (9.4\%) & 39 (24.6\%) & 159 (100\%) & \\
\hline VCUG & & & & & 0.755 \\
\hline VUR, n (\%) & $26(70.3 \%)$ & $4(10.8 \%)$ & 7 (18.9\%) & $37(100 \%)$ & \\
\hline Normal, n (\%) & $111(65.3 \%)$ & $17(10 \%)$ & $42(24.7 \%)$ & $170(100 \%)$ & \\
\hline DMSA scan & & & & & 0.194 \\
\hline APN, n (\%) & $30(60 \%)$ & $8(16 \%)$ & $12(24 \%)$ & $50(100 \%)$ & \\
\hline Normal, n (\%) & $140(69.0 \%)$ & $16(7.9 \%)$ & 47 (23.1\%) & $203(100 \%)$ & \\
\hline Serum WBC & 12,985 & 15,195 & 13,230 & & 0.666 \\
\hline Serum CRP & 2.64 & 3.17 & 2 & & 0.309 \\
\hline
\end{tabular}

Abbreviations: UTI, urinary tract infection; VCUG, voiding cystourethrogram; VUR, vesico-urethral reflux; DMSA, dimercaptosuccinic acid; APN, Acute pyelonephritis; WBC, white blood cell; CRP, C-reactive protein. 
breastfeeding and childhood hospitalization, infants $\leq 6$ months in age who were formulafed had 1.46-fold higher hospitalization risk from UTI than infants who were breastfed ${ }^{19)}$. In our study, we assessed the association between UTI and feeding patterns but could not confirm a direct relationship between them. Although the percentage of breast feeding in the UTI group (22.4\%) was lower than the value of the control group (31.3\%), the difference was not significant $(P=0.178)$. Breast milk includes various antimicrobial substances and anti-inflammatory components that protect infants from various infections and promotes tolerance and priming of the infant's immune system ${ }^{20,21)}$. However, we could not confirm a direct association between UTI and obesity in our study, and additional studies are warranted in the future.

This study is a retrospective study explaining the statistical association between obesity and UTI in a cohort of infant patients ( $<2$ years old). However, we could not identify the cause-and-effect (outcome), which is a limitation. Furthermore, subdividing the patient cohort into 3 groups based on their age reduced the sample size of the control group. Future studies with a greater number of study participants will provide more accurate outcomes regarding the association between UTI and age/obesity. A comparison of birthweight and current weight to assess the association according to the degree of weight gain, checking for the presence of large-for-gestational-age, and examining the prenatal history of the mother would have provided additional interesting findings.

In conclusion, to our knowledge, this study is the second study that confirmed the association between obesity and UTI in young children, and the first study that used febrile children without UTI as a control group. Although not all infant patients ( $\leq 2$ years old) visiting hospital for fever symptoms should undergo urine tests, we recommend that obesity should be considered a risk factor for UTI in young children. Therefore, urine tests are recommended, especially for febrile children $\leq 5$ months of age, considering the association between obesity and UTI.

\section{Patient consent}

This study was approved by the institutional review board (IRB), and the consent was waived due to the nature of the retrospective study [IRB no. 2018-04-014].

\section{Conflicts of interest}

No potential conflict of interest relevant to this article was reported.

\section{References}

1. Hudson A, Romao RLP, MacLellan D. Urinary tract infection in children. CMAJ 2017;189;E608.

2. Robert K, Bonita S, Joseph St. G, Nina S. Nelson text of pediatrics 20th edition. Elsevier Science Health Science 2016:1280-7.

3. Stokland E, Hellström M, Jacobsson B, Jodal U, Sixt R. Renal damage one year after first urinary tract infection: role of dimercaptosuccinic acid scintigraphy. J Pediatr 1996;129:815-20.

4. Kim YB, Tang CL, Koo JW. Is vaginal reflux associated with urinary tract infection in female children under the age of 36 months? Korean J Pediatr 2018;61:17-23.

5. Ellis D. Avner, William E. Harmon, Patrick Niaudet, Norishige Yoshikawa. Pediatric Nephrology 6th edition. Springer Berlin Heidelberg 2009:1007-25.

6. Mydlo JH. The impact of obesity in urology. Urol Clin North Am 2004;31:275-87.

7. Yang $T H$, Yim HE, Yoo KH. Obesity and a febrile urinary tract infection: Dual burden for young children? Urology 2014;84:445-9.

8. McCormick DP, Sarpong K, Jordan L, Ray LA, Jain S. Infant obesity: are we ready to make this diagnosis? J Pediatr 2010;157:15-9.

9. Dietz WH. The obesity epidemic in young children. Reduce television viewing and promote playing. BMJ 2001;322:313-4.

10. Twells $L$, Newhook LA. Can exclusive breastfeeding reduce the likelihood of childhood obesity in some regions of Canada? Can J Public Health 2010;101:36-9.

11. Oddy WH. Infant feeding and obesity risk in the child. Breastfeed Rev 2012;20:7-12.

12. Newburg DS, Woo JG, Morrow AL. Characteristics and potential functions of human milk adiponectin. J Pediatr 2010;156:41-6.

13. Semins MJ, Shore AD, Makary MA, Weiner J, Matlaga BR. The impact of obesity on urinary tract infection risk. Urology 2012;79: 266-9.

14. Cooper CS, Storm DW. Infection and inflammation of the pediatric genitourinary tract. Campbell-Walsh Urology, 11th edition. Philadelphia: Elsevier, 2016:2926.

15. Spencer JD, Schwaderer AL, Becknell B, Watson J, Hains DS. The innate immune response during urinary tract infection and pye- 
www.chikd.org

lonephritis. Pediatr Nephrol 2014;29:1139-49.

16. Grier WR, Kratimenos P, Singh S, Guaghan JP, Koutroulis I. Obesity as a Risk Factor for Urinary Tract Infection in Children. Clin Pediatr 2016:55:952-6.

17. Yim HE, Ha KS, Bae IS, Yoo KH, Hong YS, Lee JW. Overweight, hypertension and renal dysfunction in adulthood of neonatally overfed rats. J Nutr Biochem 2013;24:1324-33.

18. Yim HE, Yoo KH, Bae IS, Hong YS, Lee JW. Postnatal early over nutrition causes long-term renal decline in aging male rats. Pediatr
Res 2014;7:259-65.

19. Ajetunmobi OM, Whyte B, Chalmers J, Tappin DM, Wolfson L, Fleming $M$, et al. Breastfeeding is associated with reduced childhood hospitalization: evidence from a Scottish Birth Cohort (1997-2009). J Pediatr 2015;166:620-5.

20. Blewett HJH, Cicalo MC, Holland CD, Field CJ. The immunological components of human milk. Adv Food Nutr 2018;54:45-80.

21. Hanson LA, Korotkova M, Lundin S. The transfer of immunity from mother to child. Ann NY Acad Sci 2010;987:199-206. 